\title{
Zeeman Effect of Sm Atoms by High-Resolution Diode-Laser Spectroscopy
}

\author{
Wei-Guo Jin and Tatsuya Minowa \\ Department of Physics, Faculty of Science, Toho University, Miyama 2-2-1, Funabashi, Chiba 274-8510, Japan \\ Correspondence should be addressed to Wei-Guo Jin; jin@ph.sci.toho-u.ac.jp
}

Received 28 November 2012; Accepted 30 April 2013

Academic Editor: Gong Ru Lin

Copyright ( 2013 W.-G. Jin and T. Minowa. This is an open access article distributed under the Creative Commons Attribution License, which permits unrestricted use, distribution, and reproduction in any medium, provided the original work is properly cited.

High-resolution atomic-beam diode-laser spectroscopy in Sm I has been performed. Zeeman spectra have been measured for the three optical transitions at different external magnetic fields and well resolved at the magnetic fields of stronger than $6.0 \mathrm{mT}$. Using the known precise Landé $g$-factors of the ground multiplet, the Landé $g$-factors of the upper $4 f^{6} 6 s 6 p{ }^{9} F_{1}$ and ${ }^{9} D_{3}$ levels have been determined, and their precision has been improved compared with the reference values.

\section{Introduction}

Studies of the interaction of atoms with an external magnetic field, the Zeeman effect, have a long history and have been of repeated interests to spectroscopists [1-5]. The atomic Landé $g$-factor, a measure of the atomic magnetic moment, can be deduced from the Zeeman spectroscopy and provides a sensitive test of atomic calculation [6-8]. The Zeeman effect is also related to the research of the atomic parity nonconservation [9]. Moreover, precise information of magnetic fields for interstellar clouds, which is important for understanding the role played by magnetic fields in star formation, can be obtained from the Zeeman observation [10-12]. Study of the Zeeman effect is, therefore, of much interest not only from the point of view of atomic physics but also from the point of view of other fields such as astrophysics.

Samarium, a typical rare earth element, is suitable for measurements of the Zeeman effect because it has rich optical transitions and many stable isotopes. The Landé- $g$ factors of the ${ }^{7} F$ ground multiplet were precisely determined using an atomic-beam magnetic resonance $[13,14]$. Some highlying levels were measured by means of atomic-beam laser spectroscopy [15-17]. Martin et al. [18] tabulated Landé- $g$ factors for almost all known excited levels of Sm, and errors were not given.

In this paper, we report high-resolution atomic-beam diode-laser spectroscopy in Sm I around $680 \mathrm{~nm}$. Zeeman spectra at different external magnetic fields are measured and analyzed for three transitions. Using the known precise values of the Landé $g$-factor of the ground multiplet, the Landé $g$-factors of the upper levels are obtained, and results are discussed.

\section{Experiment}

The present experiment was performed using a diode-laser beam and an atomic beam. The experimental setup is shown in Figure 1 and is essentially identical to that used in our previous works $[19,20]$. Evaporation of Sm atoms was made using a resistance heating of a molybdenum oven. The oven temperature was controlled at about $900^{\circ} \mathrm{C}$ in order to produce a sufficient vapor pressure for $\mathrm{Sm}$. An atomic beam was formed by a $2 \mathrm{~mm}$ diameter aperture at a distance of $30 \mathrm{~cm}$ from the oven.

A laser beam was produced using a commercial tunable diode laser with an external cavity system (EOSI ECU201001A). The wavelength of the laser, from $665 \mathrm{~nm}$ to $690 \mathrm{~nm}$, was measured with a high-precision wavemeter. The output power was about $8 \mathrm{~mW}$ and the linewidth of the laser beam was smaller than $100 \mathrm{kHz}$.

In order to reduce the Doppler broadening, the laser beam crossed the atomic beam perpendicularly. At the interaction region, the intensity of the atomic beam with a diameter of about $2 \mathrm{~mm}$ was estimated to be about $2 \times 10^{12}$ 
TABLE 1: Wavelengths of the studied transitions and properties of the lower and upper levels in Sm I.

\begin{tabular}{lcccccc}
\hline Wavelength $(\mathrm{nm})$ & Lower level & & \multicolumn{2}{c}{ Upper level } \\
Level & Configuration & Energy $\left(\mathrm{cm}^{-1}\right)$ \\
\hline 672.59 & $4 f^{6} 6 s^{2}$ & ${ }^{7} F_{0}$ & 0.0 & $4 f^{6} 6 s 6 p$ & ${ }^{9} F_{1}$ & 14863.85 \\
680.30 & $4 f^{6} 6 s^{2}$ & ${ }^{7} F_{2}$ & 811.92 & $4 f^{6} 6 s 6 p$ & ${ }^{9} D_{3}$ & 15507.35 \\
686.09 & $4 f^{6} 6 s^{2}$ & ${ }^{7} F_{1}$ & 292.58 & $4 f^{6} 6 s 6 p$ & ${ }^{9} F_{1}$ & 14863.85 \\
\hline
\end{tabular}

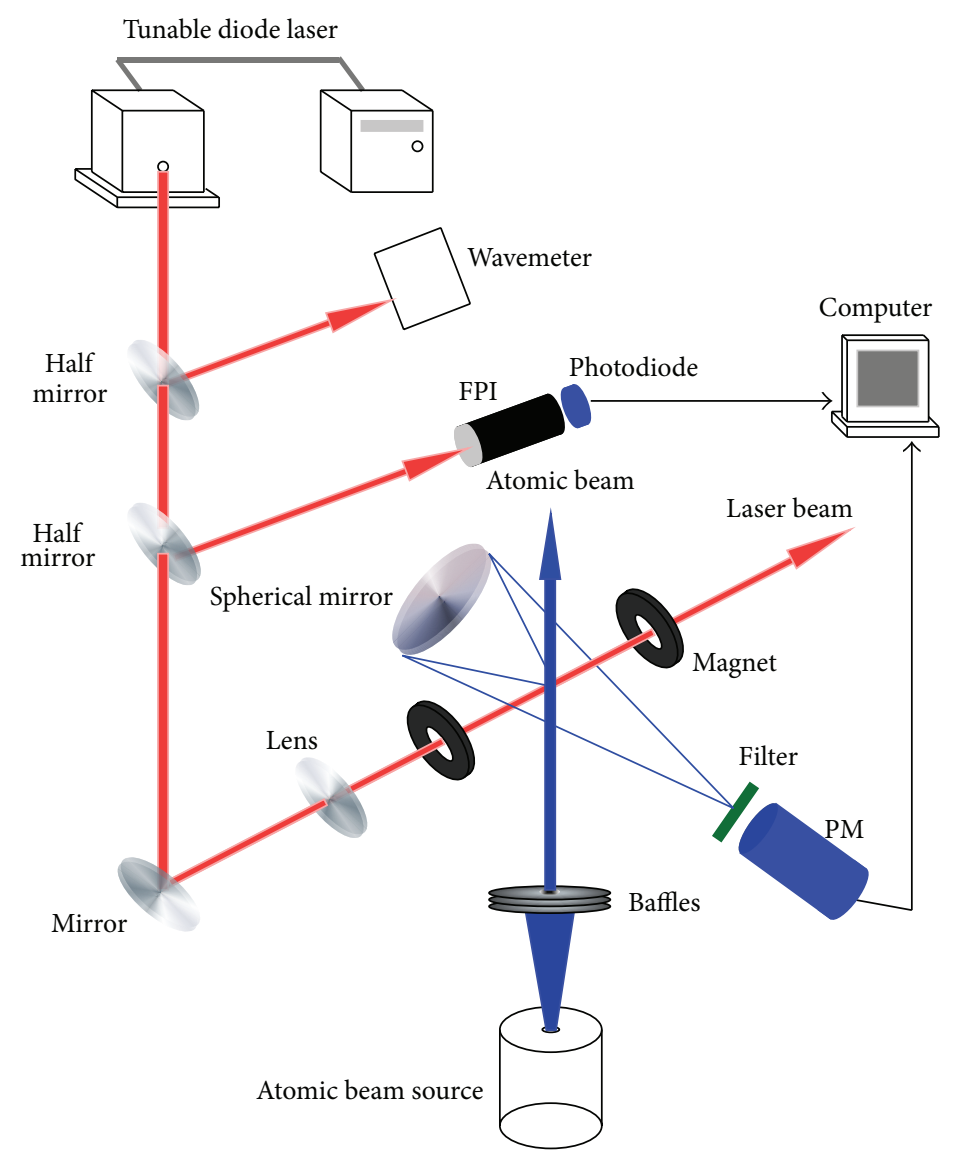

FIGURE 1: Experimental setup. FPI denotes Fabry-Perot interferometer and PM photomultiplier.

atoms/s from the oven temperature using the equation given by Ramsey [21], and the intensity of the laser beam with a diameter of about $3 \mathrm{~mm}$ was estimated to be about $3 \times$ $10^{15}$ photons/s. Fluorescence from the atomic beam, induced by the laser beam, was collected with a spherical mirror and detected with a cooled photon-counting photomultiplier (Hamamatsu R2257P). Relative frequency calibration was made by measuring the spectrum of transmitted light through a confocal Fabry-Perot interferometer (FPI) with a free spectral range of $300 \mathrm{MHz}$.

The magnetic field at the interaction region between the laser beam and the atomic beam was produced using a pair of permanent magnets. The magnets had a ring shape with an inner diameter of 13-32 mm, an outer diameter of 40-60 mm, and a thickness of $4.9-8.2 \mathrm{~mm}$, and the magnetic field could be changed using different magnets. The magnets were set along the laser beam and, therefore, the magnetic field was almost parallel to the laser beam, that is, perpendicular to the atomic beam.

\section{Results and Analysis}

Three transitions in Sm I were studied in this experiment. Wavelengths of the transitions, electronic configurations, and energies of the lower and upper levels $[18,22]$ are presented in Table 1. All transitions are from the levels of the ground term $4 f^{6} 6 s^{2}{ }^{7} F$ to the levels of the $4 f^{6} 6 s 6 p$ configuration, that is, the $s^{2}-s p$ transitions. For the $672.59 \mathrm{~nm}$ and $686.09 \mathrm{~nm}$ transitions, the upper levels are of a same level of $4 f^{6} 6 s 6 p{ }^{9} F_{1}$.

Figure 2 shows a typically observed fluorescence spectrum for the $680.30 \mathrm{~nm}$ transition in Sm I. Stable Sm has five even-mass isotopes, ${ }^{144} \mathrm{Sm},{ }^{148} \mathrm{Sm},{ }^{150} \mathrm{Sm},{ }^{152} \mathrm{Sm}$, and ${ }^{154} \mathrm{Sm}$, and two odd-mass isotopes, ${ }^{147} \mathrm{Sm}$, and ${ }^{149} \mathrm{Sm} .{ }^{147} \mathrm{Sm}$ and ${ }^{149} \mathrm{Sm}$, both, with the nuclear spin of $I=7 / 2$, have 


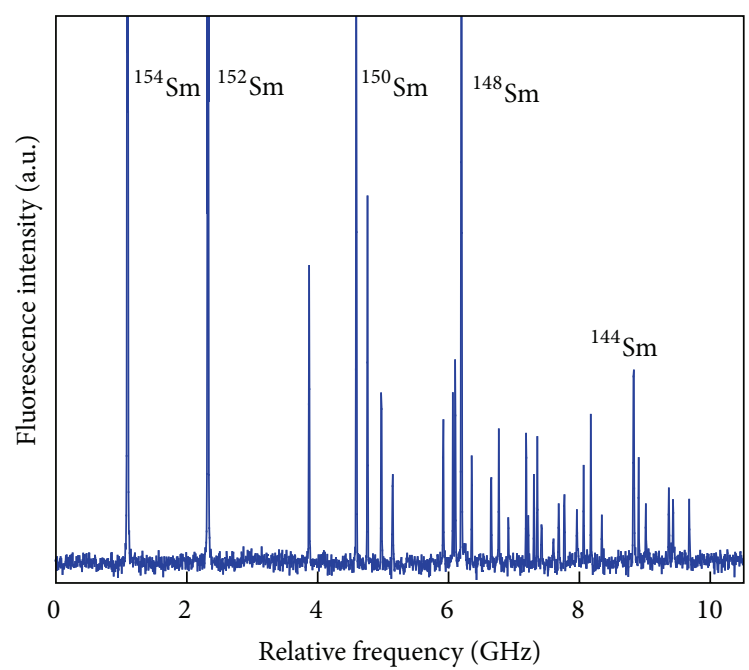

FIGURE 2: Observed fluorescence spectrum of the $4 f^{6} 6 s^{2}{ }^{7} F_{2}-$ $4 f^{6} 6 s 6 p{ }^{9} D_{3}$ transition at $680.30 \mathrm{~nm}$ in Sm I. Peaks of the evenmass isotopes are labeled with their atomic symbol. Other peaks are hyperfine structure peaks of the two odd-mass isotopes ${ }^{147} \mathrm{Sm}$ and ${ }^{149} \mathrm{Sm}$.

complicated hyperfine structures. No hyperfine structures exist for the even-mass isotopes because they have no nuclear spin: the even-mass isotopes are suitable for studying the Zeeman effect. It can be seen from Figure 2 that there are no other peaks around the peaks of ${ }^{152} \mathrm{Sm}$ and ${ }^{154} \mathrm{Sm}$. Therefore, spectra of ${ }^{152} \mathrm{Sm}$ and ${ }^{154} \mathrm{Sm}$ were used for measurement of the Zeeman effect in this experiment.

Zeeman spectra were observed at three different magnetic fields. The observed Zeeman spectra of ${ }^{152} \mathrm{Sm}$ and ${ }^{154} \mathrm{Sm}$ are shown in Figure 3 for the $680.30 \mathrm{~nm}$ transition. Since the Zeeman spectra were observed with the permanent magnets, and the fluorescence spectra were observed without the permanent magnets, starting of the frequency scanning was different for these two spectra as shown in Figures 2 and 3. Splittings of the spectra become larger as magnetic fields become stronger. At the magnetic field of $4.1 \mathrm{mT}$, the spectra overlap considerably while the spectra become well resolved at the magnetic fields of stronger than $6.0 \mathrm{mT}$. The spectra of ${ }^{154} \mathrm{Sm}$ show a same pattern as those of ${ }^{152} \mathrm{Sm}$. The full width at half maximum (FWHM) of the peaks is about $14 \mathrm{MHz}$. This width is considered to be due to the natural width of the upper level of the transition and the residual Doppler broadening of the atomic beam since the linewidth of the laser is smaller than $100 \mathrm{kHz}$.

Peak centers of the measured spectra were determined from a least-squares fit with a Lorentz function and calibrated with the FPI spectra. For each transition, measurements were performed more than 10 times. Thus, relative differences between different peaks were determined with the uncertainty of about $0.5 \mathrm{MHz}$, which includes the error of peakcenter determination, the error of the free spectral range of the FPI $(0.046 \mathrm{MHz})$, and the error of linearity correction for frequency scanning.

For atoms in an external magnetic field $B$, the atomic level with an electronic angular momentum $J$ splits into $(2 J+1)$

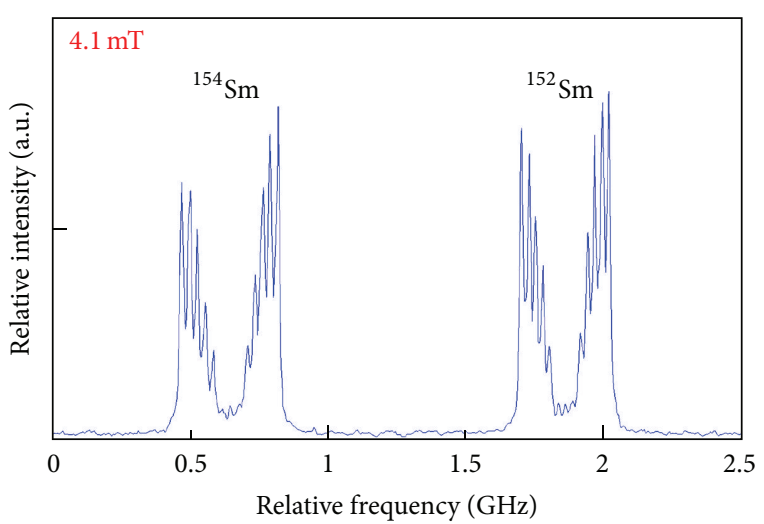

(a)

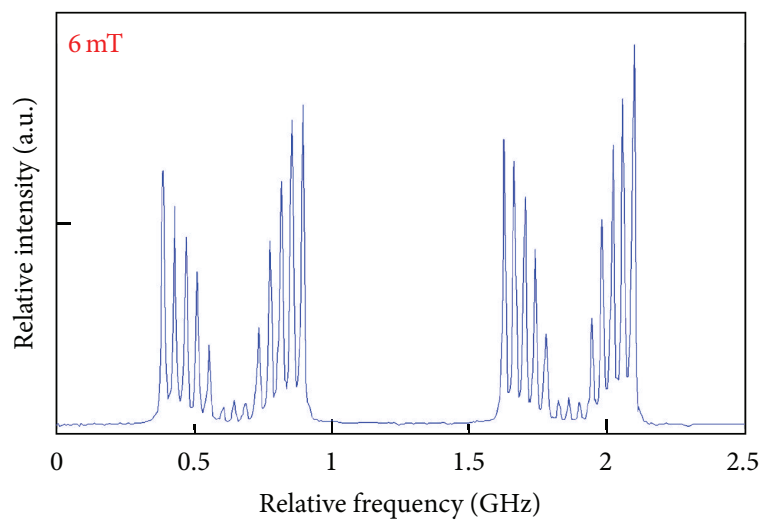

(b)

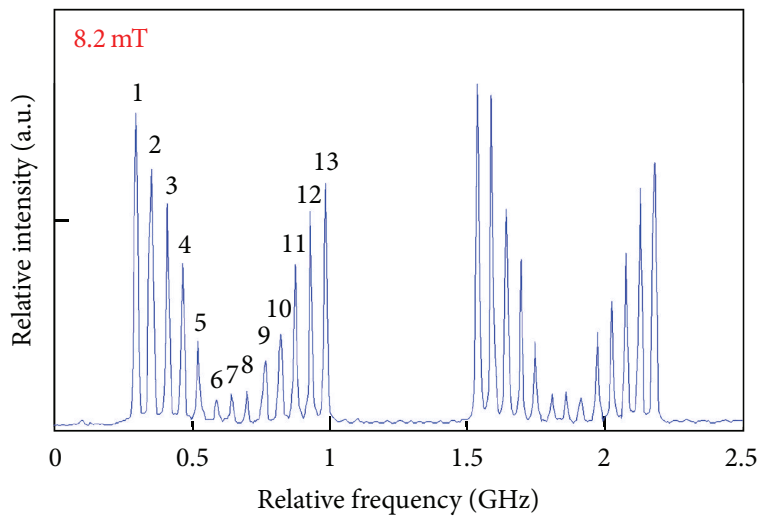

(c)

Figure 3: Observed Zeeman spectra of ${ }^{152} \mathrm{Sm}$ and ${ }^{154} \mathrm{Sm}$ at the $680.30 \mathrm{~nm}$ transition for three magnetic fields. The numbers labeled on peaks correspond to the numbers on Zeeman transitions in Figure 4.

magnetic sublevels with a magnetic quantum number $m_{J}$. The Zeeman energy $\Delta E$ from the interaction between the external weak magnetic field and the atomic magnetic moment is expressed as follows [23]:

$$
\Delta E=m_{J} g_{J} \mu_{B} B
$$

where $g_{J}$ is the atomic Landé $g$-factor and $\mu_{B}$ is the Bohr magneton. 


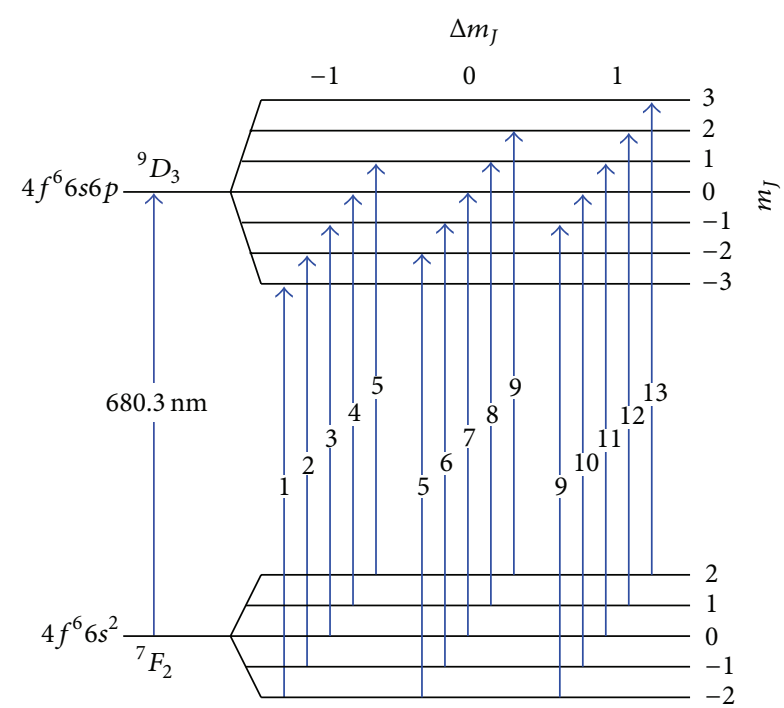

FIGURE 4: Zeeman splittings and transitions for the $680.30 \mathrm{~nm}$ transition. $m_{J}$ is the magnetic quantum number and Zeeman transitions are divided into the three series of $\Delta m_{J}=-1,0,+1$. The numbers on the Zeeman transitions correspond to the numbers labeled on peaks in Figure 3.

TABLE 2: Determined atomic Landé $g$-factors, $g_{J}$, for the $4 f^{6} 6 s 6 p{ }^{9} F_{1}$ and ${ }^{9} D_{3}$ levels in Sm I together with the reference values.

\begin{tabular}{lcccc}
\hline \multirow{2}{*}{ Magnetic Field (mT) } & \multicolumn{2}{c}{${ }^{9} F_{J}$} & \multicolumn{2}{c}{${ }^{9} \mathrm{D}_{3}$} \\
& ${ }^{154} \mathrm{Sm}$ & ${ }^{152} \mathrm{Sm}$ & ${ }^{154} \mathrm{Sm}$ & ${ }^{152} \mathrm{Sm}$ \\
\hline 6.0 & $3.097(3)$ & $3.094(3)$ & $1.946(3)$ & $1.946(3)$ \\
8.2 & $3.094(3)$ & $3.087(3)$ & $1.955(3)$ & $1.956(3)$ \\
Average & $3.093(2)$ & $1.951(3)$ \\
Reference [18] & 3.10 & \multicolumn{2}{c}{1.965} \\
\hline
\end{tabular}

For the $680.30 \mathrm{~nm}$ transition, the lower $4 f^{6} 6 s^{2}{ }^{7} F_{2}$ level with $J=2$ splits into the 5 sublevels, and the upper $4 f^{6} 6 s 6 p{ }^{9} D_{3}$ level with $J=3$ splits into the 7 sublevels. The Zeeman splittings of the $680.30 \mathrm{~nm}$ transition are shown in Figure 4 together with the possible transitions according to the selection rule of $\Delta m_{J}=m_{J}$ (the upper ${ }^{9} D_{3}$ level) $-m_{J}$ (the lower ${ }^{7} F_{2}$ level) $=0, \pm 1$.

The observed Zeeman spectra were identified with the Zeeman transitions as shown in Figure 4. For the $680.30 \mathrm{~nm}$ transition, the observed spectra shown in Figure 3 can be divided into three series of $\Delta m_{J}=-1,0,+1$. The peak intensities of the $\Delta m_{J}=-1$ series are comparable with those of the $\Delta m_{J}=+1$ series. Peaks of the $\Delta m_{J}=0$ series are also observed with lower intensities, and two of them are overlapped with those of the $\Delta m_{J}= \pm 1$ series.

From the observed Zeeman spectra, Zeeman splitting energies of both the lower and the upper levels can be obtained and are related to the external magnetic field and the $g_{J}$ values of the lower and upper levels as shown in (1). Using the known precise $g_{I}$ values of the ${ }^{7} F_{1}$ and ${ }^{7} F_{2}$ levels, the external magnetic fields $B$ were calibrated from the measured Zeeman splitting energies of the lower levels. Since the $g_{J}$ values of the lower levels were precisely measured, the uncertainty of the external magnetic field was determined by the uncertainty of the measured Zeeman splitting energies (about $0.5 \mathrm{MHz}$ ) to be about $0.1-0.2 \%$. Further, the $g_{I}$ values of the upper levels were determined and are presented in Table 2. The $g_{J}$ values were derived from the spectra at the magnetic fields of $6.0 \mathrm{mT}$ and $8.2 \mathrm{mT}$; data at the magnetic field of $4.1 \mathrm{mT}$ were not used because the spectra are overlapped considerably. The uncertainty of the $g_{J}$ values of the upper levels was determined by the uncertainties of the measured Zeeman splitting energies and the calibrated magnetic field and is about $0.1-0.2 \%$ as shown in Table 2.

It can be seen from Table 2 that the $g_{J}$ values at the magnetic field of $6.0 \mathrm{mT}$ agree with the values at the magnetic field of $8.2 \mathrm{mT}$ at the margin of experimental uncertainties. The $g_{J}$ values of ${ }^{154} \mathrm{Sm}$ are in good agreement with those of ${ }^{152} \mathrm{Sm}$ and this shows that the Lande $g$-factor is independent of the isotope. Thus, the averaged $g_{J}$ values were obtained for the $4 f^{6} 6 s 6 p{ }^{9} F_{1}$ and ${ }^{9} D_{3}$ levels. The $g_{J}$ values tabulated by Martin et al. [18] are also listed in Table 2 for comparison. The reference $g_{I}$ values are very close to the present values. Because errors are not given for the reference $g_{J}$ values, it is difficult to make detailed comparison between the present values and the reference values. For the $4 f^{6} 6 s 6 p{ }^{9} F_{1}$ level, the precision of the present $g_{J}$ value is, however, improved at least one order of magnitude compared with the reference value.

\section{Summary}

High-resolution laser spectroscopy in Sm I has been performed using the diode-laser beam together with the collimated atomic beam. Fluorescence spectra have been observed for the three optical transitions, and the spectra of ${ }^{152} \mathrm{Sm}$ and ${ }^{154} \mathrm{Sm}$ have been used for measurements of the Zeeman effect. The Zeeman spectra have been measured at the three external magnetic fields and well resolved at the magnetic fields of stronger than $6.0 \mathrm{mT}$. The observed spectra have been identified with the Zeeman transitions and the magnetic fields have been calibrated with the known precise $g_{J}$ values of the ground multiplet. The $g_{J}$ values of the upper $4 f^{6} 6 s 6 p{ }^{9} F_{1}$ and ${ }^{9} D_{3}$ levels have been determined and show no isotopic dependence. The precision of the present $g_{J}$ values is improved compared with the reference values. The precise $g_{J}$ values of various atomic levels are useful for astrophysics.

\section{Acknowledgments}

The authors thank Mr. K. Moronaga and Dr. M. Kawamura for their help in this experiment.

\section{References}

[1] T. Jeong, J. Y. Won, and H.-R. Noh, "Line shapes in polarization spectroscopy for the rubidium $D_{1}$ line in an external magnetic field," Optics Communications, vol. 292, pp. 106-110, 2013. 
[2] G. Sinuco-León and B. M. Garraway, "Radio-frequency dressed atoms beyond the linear Zeeman effect," New Journal of Physics, vol. 14, Article ID 123008, 12 pages, 2012.

[3] J.-C. Pain and F. Gilleron, "Characterization of anomalous Zeeman patterns in complex atomic spectra," Physical Review A, vol. 85, Article ID 033409, 14 pages, 2012.

[4] J. Li, C. Dong, P. Jönsson, and G. Gaigalas, “ $M_{F}$-dependent hyperfine induced transition rates in an external magnetic field for Be-like ${ }^{47} \mathrm{Ti}^{18+}$," Physics Letters A, vol. 375, no. 5, pp. 914-917, 2011.

[5] D. Shulyak, A. Reiners, S. Wende, O. Kochukhov, N. Piskunov, and A. Seifahrt, "Modelling the molecular Zeeman-effect in Mdwarfs: methods and first results," Astronomy and Astrophysics, vol. 523, article A37, 12 pages, 2010.

[6] R. Soria Orts, J. R. Crespo López-Urrutia, H. Bruhns et al., "Zeeman splitting and $g$ factor of the $1 s^{2} 2 s^{2} 2 p^{2} P_{3 / 2}$ and ${ }^{2} P_{1 / 2}$ levels in $\mathrm{Ar}^{13+}$, Physical Review A, vol. 76, Article ID 052501, 7 pages, 2007.

[7] Y. Zhang, J. Xu, W. Zhang et al., "Lifetime and Landé factor measurements of $5 p 7 p$ levels of Sn I by time-resolved laser spectroscopy," Physical Review A, vol. 78, no. 2, Article ID 022505, 6 pages, 2008.

[8] D. L. Moskovkin, V. M. Shabaev, and W. Quint, "Zeeman effect of the hyperfine-structure levels in lithiumlike ions," Physical Review A, vol. 77, no. 6, Article ID 063421, 14 pages, 2008.

[9] E. J. Angstmann, T. H. Dinh, and V. V. Flambaum, "Parity nonconservation in atomic Zeeman transitions," Physical Review A, vol. 72, no. 5, Article ID 052108, 5 pages, 2005.

[10] R. M. Crutcher, B. Wandelt, C. Heiles, E. Falgarone, and T. H. Troland, "Magnetic fields in interstellar clouds from zeeman observations: inference of total field strengths by Bayesian analysis," The Astrophysical Journal, vol. 725, no. 1, pp. 466-479, 2010.

[11] O. Kochukhov, "The Paschen-Back effect in the Li I $6708 \AA$ line and the presence of lithium in cool magnetic Ap stars," Astronomy and Astrophysics, vol. 483, no. 2, pp. 557-566, 2008.

[12] A. López Ariste, S. Tomczyk, and R. Casini, "Hyperfine structure as a diagnostic of solar magnetic fields," The Astrophysical Journal, vol. 580, no. 1, pp. 519-527, 2002.

[13] F. M. J. Pichanick and G. K. Woodgate, "Zeeman effect in the ground multiplet of samarium," Proceedings of the Royal Society A, vol. 263, pp. 89-100, 1961.

[14] W. J. Childs and L. S. Goodman, "Reanalysis of the hyperfine structure of the $4 f^{6} 6 \mathrm{~s}^{2}{ }^{7} \mathrm{~F}$ multiplet in ${ }^{147,149} \mathrm{Sm}$, including measurements for the ${ }^{7} F_{6}$ state," Physical Review A, vol. 6, no. 6, pp. 2011-2021, 1972.

[15] T. Kobayashi, I. Endo, A. Fukumi et al., "Measurement of hyperfine structure constants, g values and tensor polarizability of excited states of Sm I," Zeitschrift für Physik D, vol. 39, no. 3, pp. 209-216, 1997.

[16] A. Fukumi, I. Endo, T. Horiguchi et al., "Stark and Zeeman spectroscopies of $4 f^{6} 6 s 6 p^{7} G_{1-6}$ levels in Sm I under external electric and magnetic fields," Zeitschrift für Physik D, vol. 42, no. 4, pp. 243-249, 1997.

[17] T. Kuwamoto, I. Endo, A. Fukumi et al., "Stark and Zeeman spectroscopies of Sm I from metastable-state atomic beam produced by electric discharge," Journal of the Physical Society of Japan, vol. 67, no. 4, pp. 1213-1219, 1998.

[18] W. C. Martin, R. Zalubas, and L. Hagan, Atomic Energy Levels-The Rare-Earth Elements, NSRDS-NBS60, US GPO, Washington, DC, USA, 1978.
[19] W. G. Jin, Y. Nemoto, H. Nakai, M. Kawamura, and T. Minowa, "Isotope shifts in Gd I and Er I by UV laser spectroscopy," Journal of the Physical Society of Japan, vol. 77, no. 12, Article ID 124301, 5 pages, 2008.

[20] T. Wakui, W. G. Jin, K. Hasegawa, H. Uematsu, T. Minowa, and H. Katsuragawa, "High-resolution diode-laser spectroscopy of the rare-earth elements," Journal of the Physical Society of Japan, vol. 72, no. 9, pp. 2219-2223, 2003.

[21] N. F. Ramsey, Molecular Beams, Oxford University Press, Oxford, UK, 1985.

[22] W. F. Meggers, C. H. Corliss, and B. F. Scribner, Tables of Spectral-Line Intensities-Part I: Arranged by Elements, NBS Monograph 145, US GPO, Washington, DC, USA, 1975.

[23] H. Haken and H. C. Wolf, The Physics of Atoms and Quanta, Springer, Berlin, Germany, 1996. 

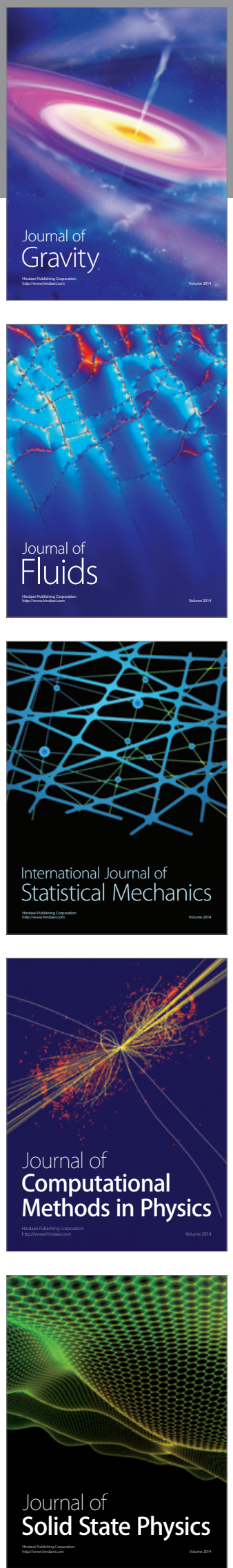

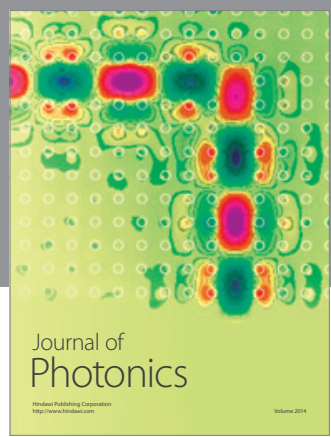

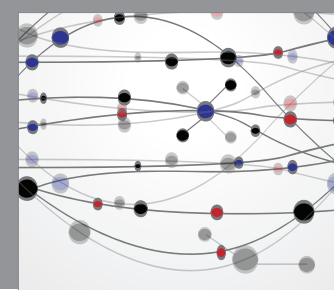

The Scientific World Journal

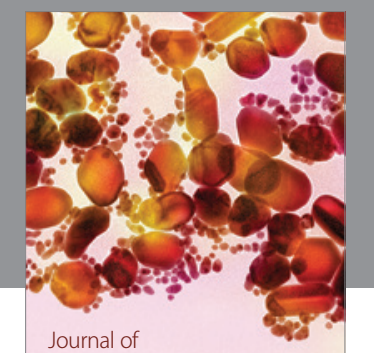

Soft Matter
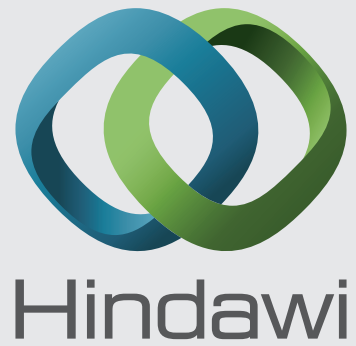

Submit your manuscripts at

http://www.hindawi.com
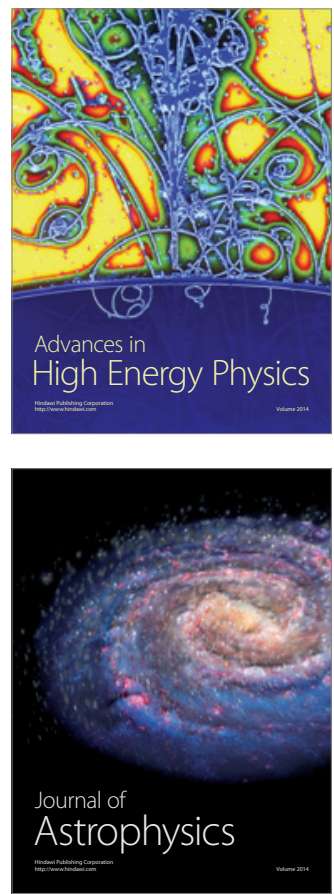
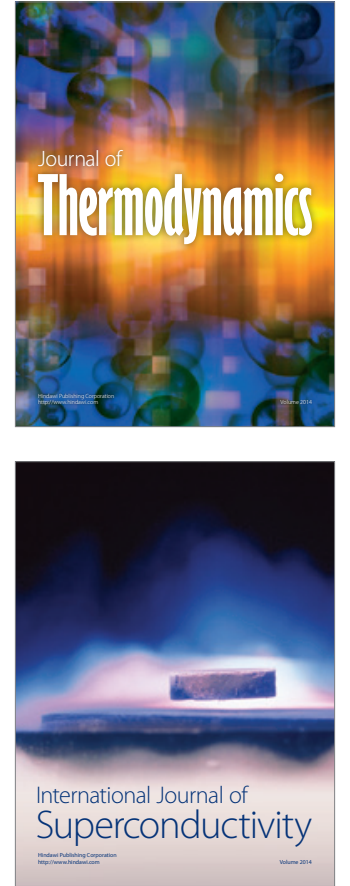
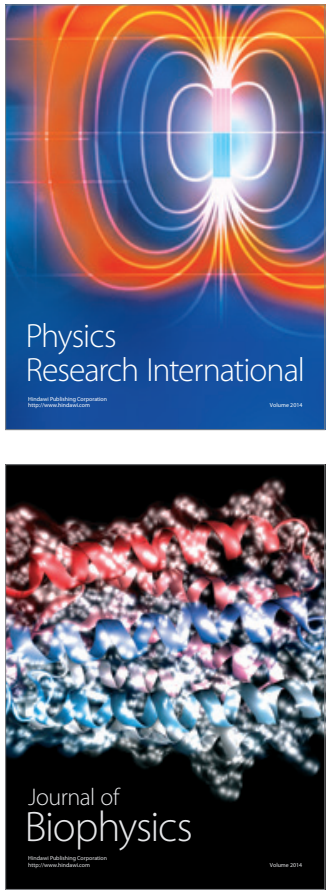
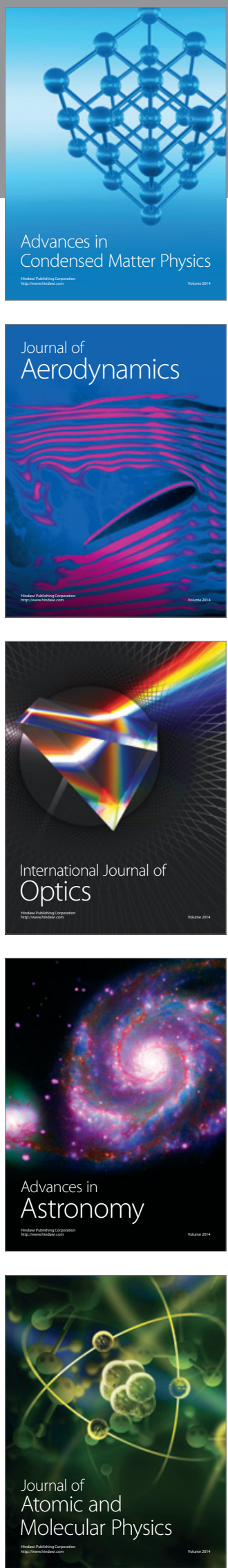